Supplementary Material

\title{
Polymorphism and Superconductivity in bilayer molecular metals $(\mathrm{CNB}-\mathrm{EDT}-\mathrm{TTF})_{4} \mathrm{I}_{3}$.
}

Sandra Rabaça, ${ }^{*[a]}$ Sandrina Oliveira, ${ }^{[a]}$ Isabel C. Santos, ${ }^{[a, b]}$ Vasco Gama,${ }^{[a]}$ Dulce Belo, ${ }^{[a]}$ Elsa B. Lopes, ${ }^{[a]}$ Enric Canadell, ${ }^{[c]}$ Manuel Almeida* ${ }^{[a]}$.

${ }^{[a]} \mathrm{C}^{2} \mathrm{TN}$, Instituto Superior Técnico, Universidade de Lisboa Estrada Nacional 10, P-2695-066 Bobadela LRS, Portugal

${ }^{[b]}$ CQE, Instituto Superior Técnico, Universidade de Lisboa Av. Rovisco Pais, P-1049-001 Lisboa, Portugal

${ }^{[c]}$ Institut de Ciencia de Materials de Barcelona (ICMAB-CSIC), Campus UAB, E-08193 Bellaterra, Spain.

KEYWORDS Molecular metals, Metallic bilayers, Cyanobenzene-TTF derivatives, Organic superconductors. 


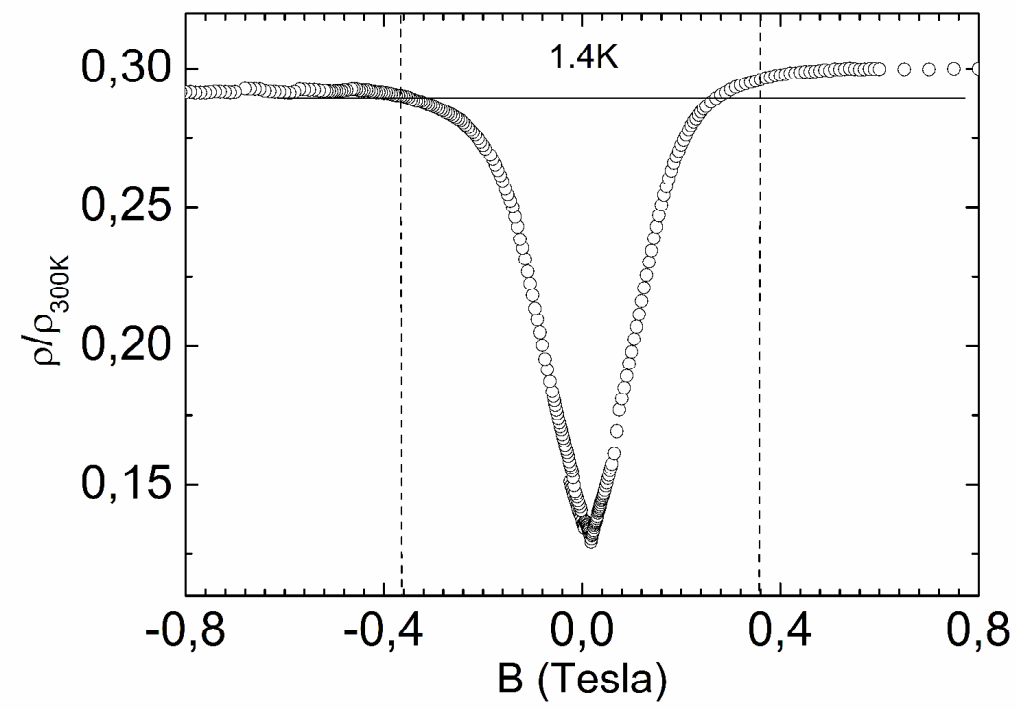

Figure S1. Variation of the normalized resistivity of the $k$-(CNB-EDT-TTF $)_{4} \mathrm{I}_{3}$ as a function of magnetic field at $1.4 \mathrm{~K}$, where it is possible to see the low critical field of the superconducting transition. 